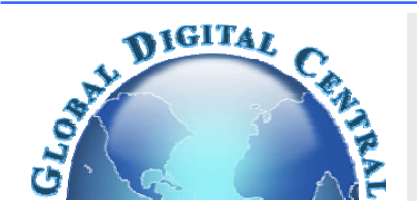

\author{
Frontiers in Heat and Mass Transfer
}

Available at www. ThermalFluidsCentral.org

\title{
IMPACT OF CATTANEO-CHRISTOV HEAT FLUX IN THE CASSON FLUID FLOW OVER A STRETCHING SURFACE WITH ALIGNED MAGNETIC FIELD AND HOMOGENEOUS - HETEROGENEOUS CHEMICAL REACTION
}

\author{
P. Bala Anki Reddy ${ }^{a^{*}}$ and S. Suneetha ${ }^{\mathrm{b}}$ \\ $a^{*}$ Department of Mathematics, School of Advanced Sciences, Vellore Institute of Technology, Vellore-632014, INDIA
}

${ }^{b}$ Department of Applied Mathematics, Yogi Vemana University, Kadapa-516003, Andhra Pradesh, INDIA

\begin{abstract}
This work concentrates on the effects of homogeneous-heterogeneous chemical reactions on MHD boundary layer flow of Casson fluid over a stretching surface. Cattaneo-Christov heat flux model is considered instead of classical Fourier's law to explore the heat transfer phenomena. Appropriate similarity transformations are used to convert the governing partial differential equations into a system of coupled non-linear differential equations. The resulting coupled non-linear differential equations are solved numerically by using the fourth order Runge-Kutta method with shooting technique. The impact of significant parameters on velocity, temperature, concentration, skin friction coefficient and the Nusselt number are presented graphically and in tabular form. Our computations disclose that fluid temperature has inverse relationship with the thermal relaxation time. Also, the strength of homogeneous and heterogeneous parameters has no effect on the skin friction coefficient and Nusselt number.
\end{abstract}

Keywords: Casson fluid, MHD, Cattaneo-Christov heat flux, stretched surface, Homogeneous-Heterogeneous reactions

\section{INTRODUCTION}

In our daily life, we come across many non-Newtonian fluids such as tomato ketchup, shampoo, sugar solutions, paints, certain oils, lubricants and so on. The analysis of non-Newtonian fluids is noteworthy because of various industrial and engineering applications. Such fluids are encountered in the process of manufacturing coated sheets, foods, drilling muds, cosmetic products, dilute polymer solutions, polymeric melts etc. For instance, if one uses a nonNewtonian fluid as the coolant or heat exchangers, the required pumping power may be substantially reduced. Therefore, the fundamental analysis of the flow field of non-Newtonian fluids in a boundary layer is a vital part in the study of fluid dynamics and heat transfer. A single constitutive equation cannot describe all the properties of non-Newtonian fluids. These fluids are complex in nature so, several non-Newtonian fluids models have been recommended such as Viscoplastic (Hassan et al. 2013), Bingham plastic (Kleppe and Marner 1972), Brinkman type (Zakaria et al. 2013), power law (Olajuwon 2009), Oldroyd-B (Khan et al. 2012) and Walter-B (Khan et al. 2014) models. This is due to the versatility of fluid characteristics in nature. Although much information is available on the boundary layer flow of viscous fluids, such trails for non- Newtonian fluids are few. Actually, in non-Newtonian fluids, the derived differential equations are more nonlinear than for a viscous fluid. To find the solution of such equations is not an easy job. In the category of non-Newtonian fluids, Casson fluid has distinct features. Motivated by the versatility of fluid characteristics the Casson fluid model has considered.
Casson (Casson 1959) introduced a non-Newtonian model which was generally known as Casson model, which is utilized to identify the non-Newtonian fluid behaviour. Examples of Casson fluid include jelly, tomato sauce, honey, concentrated fruit juices etc. In the previous literature, the Casson fluid model is occasionally stated for many materials that it fit rheological data better when compared to the general viscoplastic models. Casson fluid is elucidated as a shear thinning liquid which is supposed to have an infinite viscosity at zero rate of shear, a yield stress below which no flow occurs, and a zero viscosity at an infinite rate of shear (Dash et al. 1996). A Casson fluid exhibits a yield stress. If the applied shear stress is less than the yield stress on the fluid, it behaves like a solid, otherwise, it starts to move. When blood flows through small vessels at low shear rates, the blood flow is described by Casson fluid model. The unsteady heat transfer flow for a Casson fluid over a moving flat plate was investigated by Mustafa et al. (2011) by using homotopic method and also reported that by increasing Casson parameter the surface shear stress and surface heat transfer are improved. The non-Newtonian fluid flow over a stretching sheet has gained interest because of their extensive applications in industrial manufacturing such as wire drawing, drawing of plastic films, glass fiber, paper production, hot rolling and manufacturing of polymeric sheets. Crane (1970) was the first who describe the analytical solution for a stretching sheet in laminar boundary layer flow. The effect of increasing the Casson parameter is to suppress the velocity field and the temperature is enhanced over a nonlinearly stretching surface was reported by Mukhopadhyay (2013). Bhattacharyya (2013) explores that similar to Newtonian case, the self-similar solution is of dual nature in some situations both cases shrinking sheet and also for stretching sheet the solution is always unique in the boundary layer stagnation point

\footnotetext{
${ }^{*}$ Corresponding author. Email: pbarmaths@gmail.com
} 
flow of Casson fluid. Also, it is found that the velocity and thermal boundary layer thicknesses are larger for Casson fluid than that of Newtonian fluid. Swati Mukhopadhyay et al. (2013) investigated Casson fluid flow over an unsteady stretching surface where the rate of cooling can be increased by using the Prandtl number in conducting flows. Some researchers have examined Casson fluid flow over stretching surface.

Heat transfer dynamics is a significant fact in the nature which occurs due to temperature difference between two bodies or within the same body. The dynamics of heat transfer has enormous applications in engineering and industrial processes. For example, nuclear reactor cooling, wire drawing, cooper materials, cooling of electronic devices, heat conduction in tissues, refrigeration, heat pumps, energy production, etc. The heat transfer phenomena can interpret successfully by the Fourier's law of heat conduction law. But the unfavorable circumstance for the temperature field is that it produces a parabolic energy equation. Cattaneo (1948) introduced the thermal relaxation time in the Fourier's model in which the propagation speed of heat disturbance is finite. Later, Christov (2009) further modified the Cattaneo model by replacing the ordinary derivative with the Oldroyd's upper-convected derivative named as Cattaneo - Christov heat flux model, which successful preserves the material-invariant formulation. Mustafa (2015) suggested the uniqueness of Cattaneo-Christov heat flux in the rotating flow of Maxwell fluid over a linear stretching sheet. Further fluid temperature has inverse relationship with the relaxation time for heat flux and with the Prandtl number. Cattaneo-Christov heat flux model is imposed to disclose the heat transfer characteristics of variable thermal conductivity viscoelastic fluid over a stretching sheet with variable thickness was investigated by Hayat et al. (2015). Cattaneo-Christov heat flux model for Sisko fluid flow past a permeable non-linearly stretching cylinder was explore by Malik et al. (2016). Further, it was found that both the velocity and temperature diminished with increasing values of the power-law index and stretching parameter whereas, the temperature was found to be inversely proportional to the thermal relaxation time. Hayat et al. (2016) scrutinized the flow of Jeffrey fluid for Cattaneo-Christov heat flux Model, and found that the stretching rates ratio has opposite impact on temperature profile. More recently, researchers (Hayat et al (2016), Khan et al (2017), Tasawar Hayat et al (2018)) studied the CattaneoChristov heat flux model on different channels.

MHD problems occur in several situations like the prediction of space weather, damping of turbulent fluctuations in semiconductor melts in crystal growth, measurement of flow rates of beverages in food industry. Studies on heat transfer of non-Newtonian fluid flows on a stretching sheet with MHD find many applications in engineering and industry. For example, in the extrusion of polymers through a slit die for the manufacture of plastic sheets, the sheet is sometimes stretched. In these processes, the final product properties mainly depend on the rate of cooling. If such a sheet in an electrically conducting Casson fluid under the influence of a magnetic field is drawn, the rate of cooling can be controlled, so that the end product can be obtained with the desired quality. Bala Anki Reddy et al. (2016) examined the numerical Study of MHD Boundary Layer Slip Flow of a Maxwell Nanofluid over an exponentially stretching surface with convective boundary condition. Also, found that the thermal boundary layer thickness enhances with Brownian motion and thermophoresis parameters. Bala Anki Reddy and Bhaskar Reddy (2011) analyzed thermal radiation effects on an exponentially stretching sheet with MHD. The results obtained by Kirubhashankar (2015) on Casson fluid flow and heat transfer over an MHD unsteady porous stretching surface may be helpful for possible technological applications in liquid based systems involving stretchable materials. Bhattacharyya et al. (2013) studied the analytic solution for MHD boundary layer flow of Casson fluid over a stretching/shrinking sheet with mass transfer the magnetic field changes the behavior of the entire flow dynamics in the shrinking sheet case and also has a major influence in the stretching sheet case. Khalid et al. (2015) investigated unsteady MHD flow of Casson fluid past over an oscillating vertical plate embedded in a porous medium and found that when the Casson parameter is large enough ie., $\beta \rightarrow \infty$, the non-Newtonian behaviour disappear and the fluid purely behaves like a Newtonian fluid. Thus, the velocity boundary layer thickness for Casson fluid is larger than the Newtonian fluid. Many researchers have been attracted by Casson fluid model and discussed the boundary layer flow problem of Casson fluid with various physical effects such as magnetic field and stretching sheet [28, 29, 30, 31].

Most of the Chemical reactions involve either heterogeneous or homogeneous processes. Some reactions are very slow or not at all, except in the presence of catalyst. A complex interaction lies between the homogeneous and heterogeneous reactions which is incorporated in the production and consumption of reactant species at different rates on the fluid and also on the catalytic surfaces, such are happened in fog formation and dispersion, food processing, manufacturing of polymer production, groves of fruit trees, moisture over agricultural fields, equipment design by chemical processing, crops damage via freezing etc. Some kind of chemical reaction is observed if there is a foreign mass in air or water. During a chemical reaction heat is generated between two species (Bird et al., 2006). Generally, the reaction rate depends on the concentration of the mass itself. If the rate of reaction is directly proportional to concentration itself (Cussler 1998), then it is said to be first order. Mukhopadhyay and Gorla (2014) analysed a chemically reactive species of a Casson fluid towards an exponentially stretching surface by numerical method and examined the first order chemical reaction and resolved that by the enhancement of the Casson parameter, there is a suppress in the velocity field and the concentration is enhanced with rising Casson parameter. Micropolar fluid flow of a homogeneous-heterogeneous reactions from a permeable stretching or shrinking sheet in a porous medium was analyzed by Shaw et al. (2013). Bala Anki Reddy (2016) studied the magnetohydrodynamic flow of a Casson fluid over an exponentially inclined permeable stretching surface with thermal radiation and chemical reaction and revealed as the concentration decreases with increase in the value of chemical reaction parameter and solutal buoyancy parameter. Srinivas et al. (2014) presented an analysis which will be of special interest for clinicians who engaged in the treatment of radiation and chemical therapy for cancer and other tumor related diseases. Mariam Sheikh and Zaheer Abbas (2015) reported Homogeneous-Heterogeneous reactions of Casson fluid due to a stretching/shrinking surface with uniform suction and slip effects.

Motivated by the above-mentioned studies, the aim here is to investigate the impact of Cattaneo-Christov heat flux flow of a nonNewtonian fluid over a stretching surface. Mathematical modelling is presented using constitutive equations of a Casson fluid. An aligned magnetic field and chemical reaction effects are taken into account. The present study is structured in the following fashion. The mathematical formulation is completed in the next section. Series solutions by using a shooting technique with Runge-Kutta method of order four are developed in the subsequent sections. Then convergence analysis and discussion are presented. Important results are summarized in the last section. To the best of author's knowledge, such study has not been reported earlier in the literature.

\section{MATHEMATICAL FORMULATION}

Consider the steady two-dimensional flow of an electrically conducting and viscous incompressible Casson fluid flow over a stretching surface. The $x$-axis is taken along the stretching surface in the direction of the motion while the $y$-axis is perpendicular to the surface. The stretching surface has the linear velocity $U_{w}(x)=m x$ where $m>0$ is the stretching sheet, $m=0$ is for the static sheet and $m<0$ is for the shrinking sheet, respectively. We assume that the rheological equation of state for an isotropic and incompressible flow of a Casson fluid is as (Mukhopadhyay 2013, Pramanik 2015): 


$$
\tau_{i j}=\left\{\begin{array}{l}
2\left(\mu_{B}+P_{y} / \sqrt{2 \pi}\right) e_{i j}, \pi>\pi_{c} \\
2\left(\mu_{B}+P_{y} / \sqrt{2 \pi_{c}}\right) e_{i j}, \pi<\pi_{c}
\end{array}\right.
$$

where $\mu_{B}$ is the plastic dynamic viscosity of the non-Newtonian fluid, $P_{y}$ is the yield stress of the fluid, $\pi=e_{i j} e_{i j}, e_{i j}$ is the $(i, j)^{\text {th }}$ component of the deformation rate and $\pi_{c}$ is the critical value of this product based on the non-Newtonian model. We also consider a simple model for the interaction between a homogeneous (or bulk) and heterogeneous (on sheet) reaction involving the two-chemical species $A$ and $B$ in a boundary layer flow as stated by Chaudhary and Merkin (1995) of the following form:

$$
\begin{aligned}
& A+2 B \rightarrow 3 B, \text { rate }=k_{c} a b^{2} \\
& A \rightarrow B, \text { rate }=k_{s} a
\end{aligned}
$$

where $a$ and $b$ are concentrations of the chemical species $A$ and $B$ respectively, and $k_{c}, k_{s}$ are the rate constants. We also assume that both reaction processes are isothermal. Under these assumptions and boundary layer approximations, the governing equations can be written as:

$\frac{\partial u}{\partial x}+\frac{\partial v}{\partial y}=0$

$u \frac{\partial u}{\partial x}+v \frac{\partial u}{\partial y}=\left(1+\frac{1}{\beta}\right) \frac{\partial^{2} u}{\partial y^{2}}-\frac{\sigma B_{0}^{2}}{\rho} \sin ^{2} \xi$

$\rho c_{p}\left(u \frac{\partial T}{\partial x}+v \frac{\partial T}{\partial y}\right)=-\nabla \cdot q$

$u \frac{\partial a}{\partial x}+v \frac{\partial a}{\partial y}=D_{A} \frac{\partial^{2} a}{\partial y^{2}}-k_{c} a b^{2}$

$u \frac{\partial b}{\partial x}+v \frac{\partial b}{\partial y}=D_{A} \frac{\partial^{2} b}{\partial y^{2}}+k_{c} a b^{2}$

The corresponding boundary conditions are

$u=u_{w}(x)+L\left(1+\frac{1}{\beta}\right) \frac{\partial u}{\partial y}, v=-v_{w}, T=T_{w}, D_{A} \frac{\partial a}{\partial y}=k_{s} a, \mathrm{D}_{B} \frac{\partial b}{\partial y}=k_{s} a$

at $y=0$

$u \rightarrow u_{e}(x)=c x, v \rightarrow 0, T \rightarrow 0, a \rightarrow a_{0}, b \rightarrow 0$ as $y \rightarrow \infty$

where $u$ and $v$ are the velocity components in the $x$ and $y$ directions respectively, $\beta$ is the Casson parameter, $\sigma$ is the electrical conductivity, $B_{0}$ is the uniform magnetic field, $\rho$ is the density of the fluid, $\xi$ is the aligned angle, $D_{A}$ is the diffusive species coefficient of $A$ and $D_{B}$ is the diffusive species coefficient of $B, L$ is the velocity slip parameter, $v_{w}$ is the constant mass flux with $v_{w}<0$ for suction and $v_{w}>0$ for injection (blowing), $T$ is the temperature of the fluid, $T_{w}$ is the surface temperature of the fluid, $u_{e}$ is the free stream velocity, $c$ is the constant and $q$ is the heat flux satisfies the following relationship:

$q+\lambda\left(\frac{\partial q}{\partial t}+V . \nabla q-q . \nabla V+(\nabla . V) q\right)=-k \nabla T$

Here $\lambda$ is the relaxation time of heat flux, $k$ is the thermal conductivity of the fluid and $V$ is the velocity vector. It may be noted that when $\lambda=0$ in Eq. (9), the problem will be reduced to Fourier's heat conduction law. Eliminating q from Eqs. (5) and (9) gives: $u \frac{\partial T}{\partial x}+v \frac{\partial T}{\partial y}=2\left(\begin{array}{l}u^{2} \frac{\partial^{2} T}{\partial x^{2}}+v^{2} \frac{\partial^{2} T}{\partial y^{2}}+2 u v \frac{\partial^{2} T}{\partial x \partial y}+ \\ u \frac{\partial u}{\partial x} \frac{\partial T}{\partial x}+v \frac{\partial v}{\partial y} \frac{\partial T}{\partial y}+u \frac{\partial v}{\partial x} \frac{\partial T}{\partial y}+v \frac{\partial u}{\partial y} \frac{\partial T}{\partial x}\end{array}\right)=\frac{k}{\rho c_{p}} \frac{\partial^{2} T}{\partial y^{2}}$

Now, introducing the following

transformation

$\eta=\sqrt{\left(\frac{c}{v}\right)} y, \psi=\sqrt{c v} x f(\eta)$

$\theta=\frac{T-T_{\infty}}{T_{w}-T_{\infty}}, \phi(\eta)=\frac{a}{a_{0}}, h(\eta)=\frac{b}{a_{0}}$

Where $\eta$ is the similarity variable and $\psi(x, y)$ is the stream function satisfying $u=\frac{\partial \psi}{\partial y}, v=-\frac{\partial \psi}{\partial x}$.

Substituting (11) into the Equations (4), (6), (7) and (10), we get the following set of ordinary differential equations

$$
\begin{aligned}
& \left(1+\frac{1}{\beta}\right) f^{\prime \prime \prime}+f f^{\prime \prime}-\left(f^{\prime}\right)^{2}-M \sin ^{2} \xi f^{\prime}=0 \\
& \frac{1}{\operatorname{Pr}} \theta^{\prime \prime}+f \theta^{\prime}-\gamma\left(f f^{\prime} \theta^{\prime}+f^{2} \theta^{\prime \prime}\right)=0 \\
& \frac{1}{S c} \phi^{\prime \prime}+f \phi^{\prime}-K \phi h^{2}=0 \\
& \frac{\delta}{S c} h^{\prime \prime}+f h^{\prime}+K \phi h^{2}=0
\end{aligned}
$$

The corresponding boundary conditions are

$$
\begin{aligned}
& f(0)=S, f^{\prime}(0)=\lambda+S_{v}\left(1+\frac{1}{\beta}\right) f^{\prime \prime}(0), \theta(0)=1, \phi^{\prime}(0)=K_{s} \phi(0), \\
& \delta h^{\prime}(0)=-K_{s} \phi(0), f^{\prime}(\infty)=1, \theta(\infty)=0, \phi(\infty)=1, h(\infty)=0
\end{aligned}
$$

where the primes denote the differentiation with respect to $\eta$, $M=\frac{\sigma B_{0}^{2}}{\rho c}$ is the magnetic parameter, $D=\frac{v c_{p}}{k}$ is the Prandtl number, $\gamma=\lambda c$ is the non-dimensional thermal relaxation time, $S c=\frac{v}{D_{A}}$ is the Schmidt number, $K=\frac{k_{c} a_{0}^{2}}{c}$ is a measure of the strength of the homogeneous reaction, $\delta=\frac{D_{B}}{D_{A}}$ is the ratio of the diffusion coefficient, $S=\frac{-v_{w}}{\sqrt{c v}}$ is the suction parameter $(S=0$ represents impermeable, $S>0$ represents suction and $S<0$ represents the injection or blowing), $\lambda=\frac{m}{c}$ is the stretching parameter $(\lambda<0$ represents the shrinking surface, $\lambda>0$ represents the stretching surface and $\lambda=0$ represents the forced convection flow towards the stagnation point on a static surface, $S_{v}=L \sqrt{\frac{c}{v}}$ is the slip parameter, $K_{s}=\frac{k_{s} l \mathrm{Re}^{-\frac{1}{2}}}{D_{A}}$ is a measure of the strength of the heterogeneous reaction and $\operatorname{Re}=\frac{U_{\infty} l}{v}$ is the Reynolds number. It is assumed that the diffusion coefficients of chemical species $A$ and $B$ to be of a comparable size. This argument provides us to make further assumption that the diffusion coefficients $\mathrm{D}_{\mathrm{A}}$ and $\mathrm{D}_{\mathrm{B}}$ are equal i.e., $\delta=1$ and thus:

$g(\eta)+h(\eta)=1$

Now Esq. (14) and (15) reduces to

$\frac{1}{S c} \phi^{\prime \prime}+f \phi^{\prime}-K \phi\left(1-\phi^{2}\right)=0$

With the boundary conditions

$\phi^{\prime}(0)=K_{s} g(0), \phi(\infty)=1$. 
Also, the quantity of physical interest in this problem is the skin friction coefficient and heat transfer rate at the sheet which are defined by

$$
\begin{aligned}
& C_{f}=\frac{\tau_{w}}{\rho u_{w}^{2}}, N u_{x}=\frac{x q_{w}}{k\left(T_{w}-T_{\infty}\right)} \\
& \text { Where } \tau_{w}=\left(\mu_{B} \frac{P_{y}}{\sqrt{2 \pi_{c}}}\right)\left(\frac{\partial u}{\partial y}\right)_{y=0} \text { and } q_{w}=-k\left(\frac{\partial T}{\partial y}\right)_{y=0}
\end{aligned}
$$

Using the similarity variables in Eq. (20), we get

$\operatorname{Re}_{x}^{\frac{1}{2}} C_{f}=(1+1 / \beta) f^{\prime \prime}(0)$ and $\frac{N u_{x}}{\sqrt{\operatorname{Re}_{x}}}=-\theta^{\prime}(0)$

where $\operatorname{Re}_{x}=\frac{x U w(x)}{v}$ is the local Reynolds number.

The above Skin-friction coefficient and local Nusselt number shows that its variation depends on the variation of the factors $\left(1+\frac{1}{\beta}\right) f^{\prime \prime}(0)$ and $-\theta^{\prime}(0)$ respectively.

\section{NUMERICAL PROCEDURE}

The set of nonlinear coupled differential equations (12), (13) and (18) subject to the boundary conditions (16) and (19) are solved numerically by using Runge-Kutta fourth order technique along with shooting method. For this, we transform the non-linear ordinary differential equations (12), (13) and (18) into a system of first order differential equations as follows:

$$
\begin{aligned}
& f^{\prime}=z, z^{\prime}=p, \\
& \mathrm{p}^{\prime}=\left(\frac{\beta}{1+\beta}\right)\left(\mathrm{z}^{2}-f p+M \sin ^{2} \xi z\right), \\
& \theta^{\prime}=q, \\
& q^{\prime}=\left(\frac{\gamma f z q-f q}{\left(\frac{1}{\operatorname{Pr}}-\gamma f^{2}\right)}\right), \\
& \phi^{\prime}=r, \\
& r^{\prime}=s c\left(\mathrm{~K} \phi(1-\phi)^{2}-f r\right) .
\end{aligned}
$$

The boundary conditions (16) and (19) becomes

$$
\begin{aligned}
& f(0)=s, f^{\prime}(0)=\lambda+s_{v}\left(1+\frac{1}{\beta}\right) \omega_{1}, \omega_{1}=f^{\prime \prime}(0), \theta(0)=1, \\
& \omega_{3}=K_{s} \phi(0), \omega_{3}=\phi^{\prime}(0) .
\end{aligned}
$$

In order to integrate (22)-(24) as an initial value problem, we require values of $p(0)$ i.e., $f^{\prime \prime}(0)$ and $q(0)$ i.e., $\phi^{\prime}(0)$. But no such values are given at the boundary. So, the suitable guesses values for $f^{\prime \prime}(0)$ and $\phi^{\prime}(0)$ is chosen and then integration is carried out. The most important factor of the shooting method is to choose an appropriate finite value of $\eta_{\infty}$. In order to determine $\eta_{\infty}$ for the boundary value problem, start with some initial guess values for some particular set of physical parameters to obtain $f^{\prime \prime}(0)$ and $\phi^{\prime}(0)$. The solving procedure is repeated with another large value of $\eta_{\infty}$ until two successive values of $f^{\prime \prime}(0)$ and $\phi^{\prime}(0)$ differ only by the specified significant digit. The last value of $\eta_{\infty}$ is finally chosen to be the most appropriate value of the limit $\eta_{\infty}$ for that particular set of parameters. The value of $\eta_{\infty}$ may change for another set of physical parameters. Once the finite value of $\eta_{\infty}$ is determined, then the integration is carried out. Compare the calculated values for $f^{\prime}, \theta$ and $\phi$ at $\eta=10$ (say) with the given boundary conditions $f^{\prime}(10)=0, \theta(10)=0, \phi(10)=0$ and adjust the estimated values, $f^{\prime \prime}(0)$ and $\phi^{\prime}(0)$ to give better approximation to the solution. We take the series values for $f^{\prime \prime}(0)$ and $\phi^{\prime}(0)$ apply the fourth order Runge-Kutta integration with step size $h=0.01$ (Pramanik [42]). The above procedure is repeated until to get the results up to the desired degree of accuracy $10^{-6}$.

\section{RESULTS AND DISCUSSION}

In order to analyze the results, numerical computations have been carried out using the method described in the previous section for various values of Magnetic parameter $M$, Aligned angle $\xi$, Casson parameter $\beta$, stretching parameter $\lambda$, Prandtl number $\operatorname{Pr}$, suction parameter $S$, slip parameter $S_{v}$, thermal relaxation time $\gamma$, Schmidt number $\mathrm{Sc}$, measure of the strength of the homogeneous reaction $\mathrm{K}$, and measure of the strength of the heterogeneous reaction $K_{s}$ on velocity, temperature and species concentration distributions is presented in Figs. 1-11.

Figure 1 illustrates that the velocity decreases with the increase of magnetic parameter $M$ for both stretching and shrinking sheet. The same effect is observed for both suction and blowing. This is due to the fact that an increase in magnetic parameter signifies an enhancement of Lorentz force, thereby reducing the magnitude of the velocity. The variations in the velocity profiles for changes in the aligned angle $\xi$ are presented in Figure 2. It can be inferred from this figure, that an increase in the aligned angle diminishes the velocity. It may take place due to the reason that an increase in the aligned angle strengthens the applied magnetic field. Generally, a raise in the magnetic field produce an opposite force to the flow, called Lorentz force. This force has tendency to reduce the velocity boundary layer thickness. Also, observed the same effect for stretching and shrinking sheet.

Figure 3 depicts the variation of velocity distribution for different values of Casson parameter $\beta$. It can be noticed that the velocity of the boundary layer reduces with increasing values of Casson parameter i.e., the decreasing yield stress (the fluid behaves as Newtonian fluid when Casson parameter increases). The decreasing nature of the momentum boundary layer thickness with increasing $\beta$ is noted. However, the thermal boundary layer thickness enhances with increasing $\beta$.

The effect of the velocity slip parameter $S_{v}$ on the fluid velocity is presented in Figure 4. It can be observed that an increase in the velocity slip enhances the velocity for the shrinking sheet. With stretching of the sheet, the retracting forces reduce momentum development and cause deceleration in the boundary layer flow i.e., reverse trend is observed for the stretching and blowing cases.

Figure 5 portrays the behaviour of Prandtl number on the thermal boundary layer for opposing, assisting and steady state cases. From this plot, it is evident that the thickness of the thermal boundary layer decreases with an increase in the value of the Prandtl number. For all values of Prandtl number the wall temperature gradient is negative, which means that the heat is always transferred from the surface to the ambient fluid. Prandtl number signifies the ratio of momentum diffusivity to thermal diffusivity. Fluids with least Prandtl number will possess higher thermal conductivities (and thicker thermal boundary layer structures), so that heat can diffuse faster from the sheet faster than for higher Pr fluids (thinner boundary layers). The suitable Prandtl numbers are quite essential in the industrial processes, because they are used to control the heat transfer rate during the final product.

In Figure 6 the impact of temperature on the thermal relaxation parameter is sketched. This figure depicts that the thermal boundary layer thickness decreases as temperature increases. It is due to the fact that as we increase the thermal relaxation parameter, particles of the material require more time to transfer heat to its neighbouring particles. Specifically, we can say that for higher values of thermal relaxation parameter material shows a non-conducting behaviour which is liable in declining the temperature distribution. Further it is also noted that for 
$\gamma=0$ heat transfers promptly throughout the material. The CattaneoChristov heat flux model is reduced to Fourier's law of heat conduction when $\gamma=0$.

Figure 7 displays the effect of aligned angle on temperature profiles. It is evident from figure that the temperature overshoot with an increase in aligned angle. It is due to the fact that a raise in magnetic field parameter enhances the thermal boundary layer thickness.

The effects of the Schmidt number on the concentration are displayed in Figure 8. It is noted that for larger Schmidt number there is an increase in the behaviour of concentration profile for stretching case and a contradiction is observed for shrinking case. The Schmidt number Sc is the ratio of a viscous diffusion rate to a molecular diffusion rate. From this figure, it is observed that for a fixed molecular diffusion rate, the enhance in Schmidt number is to enhance the viscous diffusion of the reactants rate, which reduces the fluid velocity and leads to increase the concentration of reactants.

Figures 9 and 10 elucidate the variations of homogeneous and heterogeneous reactions $(\mathrm{K}, \mathrm{Ks})$ on the concentration profile. We infer from these figures that there is a decrease in concentration when $\mathrm{K}$ and Ks increased. As more chemical reactants are consumed during the strengths of homogeneous and heterogeneous reactions are increased which causes the concentration profile to decrease. The concentration boundary layer of the reactants is growing with $\eta$ in both cases and after a definite value of $\eta$, the homogeneous and heterogeneous reactions have no effects on the concentration of the reactants i.e. they all coincide. Because near the surface homogeneous and heterogeneous reactions reduces the concentration profile but away from the surface with an increase in $\mathrm{K}$ and $\mathrm{Ks}$ diffusion reduces and less diffused particles enhance the concentration.

Figure 11 represents the effect of aligned angle on concentration profiles. It is evident from figure that increase in aligned angle slightly decreases the concentration profiles of the fluid. This parameter help to reduce the concentration boundary layer thickness. A slight decrement is observed in the concentration boundary layer of the reactants with $\eta$ and after a certain value of $\eta$, the aligned angle has no effect on the concentration of the reactants i.e. they all coexist.

Table 1 shows the comparative values of skin friction coefficient and Sherwood number for various values of $\beta, M, \xi, P r, \gamma, \lambda, S_{v}, K s, K$ and $S$. Magnitude of $f^{\prime \prime}(0)$ related to skin-friction coefficient and $g(0)$ Sherwood number decreases with increasing Casson parameter $\beta$, magnetic parameter $\mathrm{M}$ and aligned angle $\xi$. The thermal relaxation parameter $\gamma$ has no effect on $f^{\prime \prime}(0)$ but it two fold the values of $g(0)$. The stretching parameter $\lambda$ has inverse relationship with $f^{\prime \prime}(0)$ and proportional to $\mathrm{g}(0)$. An increase in the velocity slip parameter $S_{v}$ increases $f^{\prime \prime}(0)$ and a slight decrease is observed with $g(0)$. The strength of homogeneous and heterogeneous parameters has no effect on the skin friction coefficient and Sherwood number. The suction parameter $\mathrm{S}$ is proportional to both $f^{\prime \prime}(0)$ and $g(0)$.

\section{CLOSING REMARKS}

We explore the properties of the Cattaneo-Christov heat flux model for a two-dimensional hydrodynamic boundary layer flow of a Casson fluid flow over a stretching surface. Impacts of homogeneous and heterogeneous reactions are also accounted. The key points are listed below:

- The velocity field decreases with an increase in magnetic field, aligned angle and casson parameter.

- Increasing values of Prandtl number, thermal relaxation parameter corresponds to low temperature. A reverse trend is observed for aligned angle.

- Increasing the values of the strength of homogeneous and heterogeneous parameters and aligned angle decrease the wall concentration.
- $\quad$ The strength of homogeneous and heterogeneous parameters has no effect on the skin friction coefficient and Nusselt number.

- The present consideration for the Newtonian fluid case be recovered by choosing $\gamma=0$.

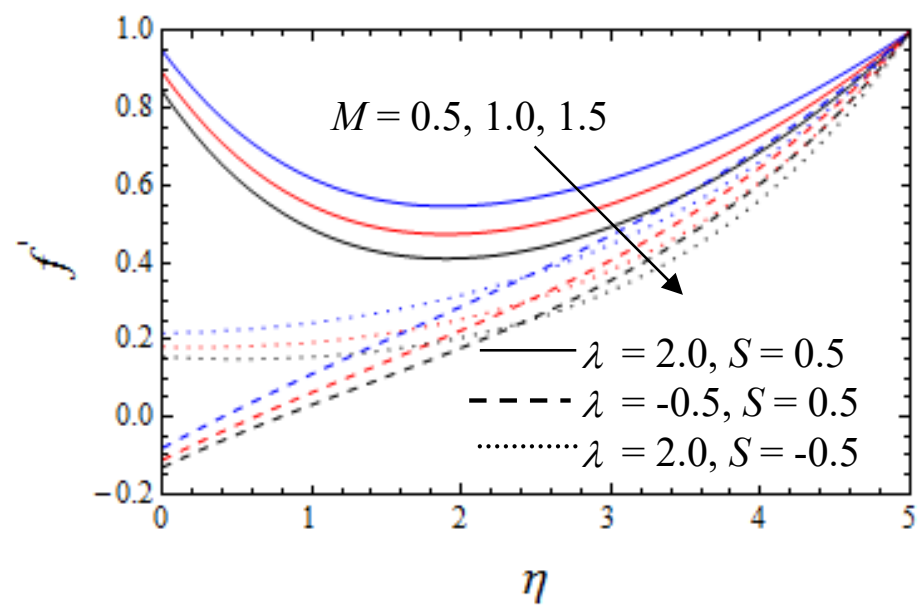

Fig. 1 Velocity profiles for $M$

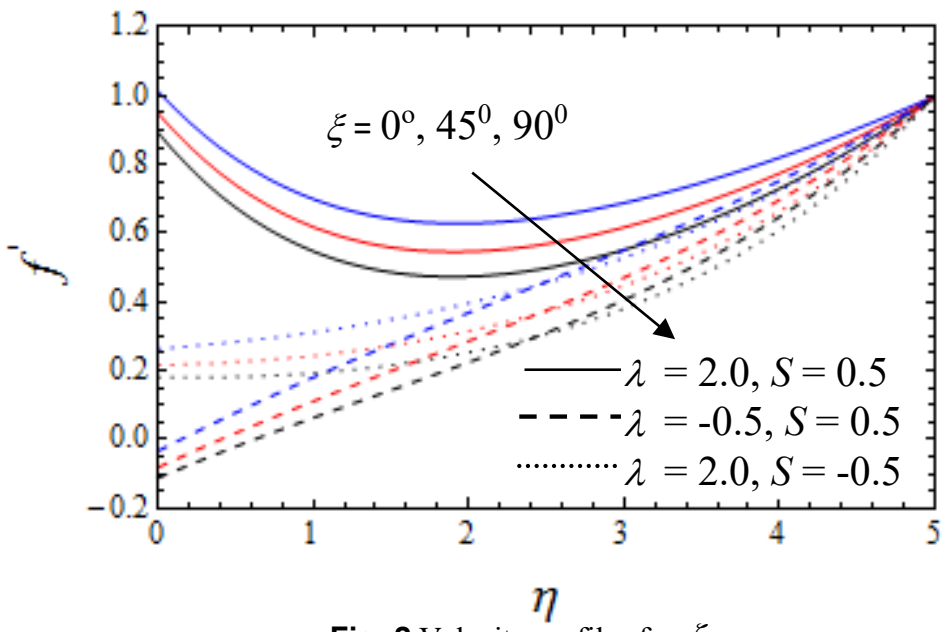

Fig. 2 Velocity profiles for $\xi$

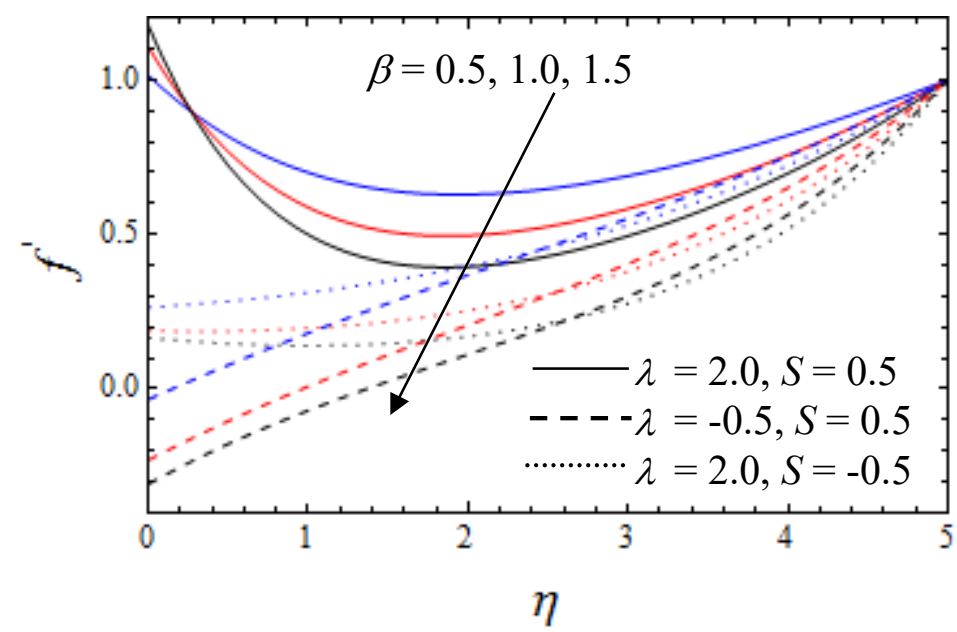

Fig. 3 Velocity profiles for $\beta$ 


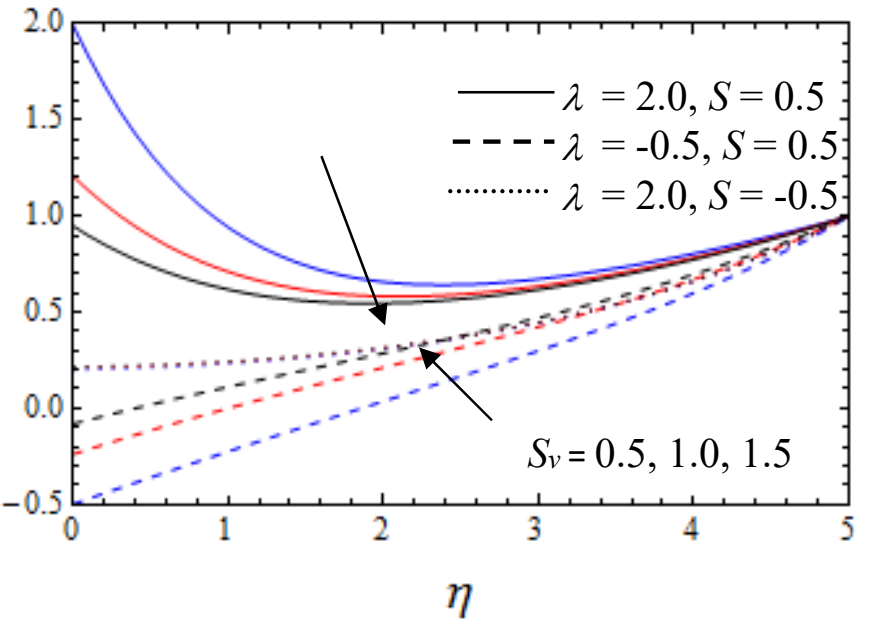

Fig. 4 Velocity profiles for $S_{v}$

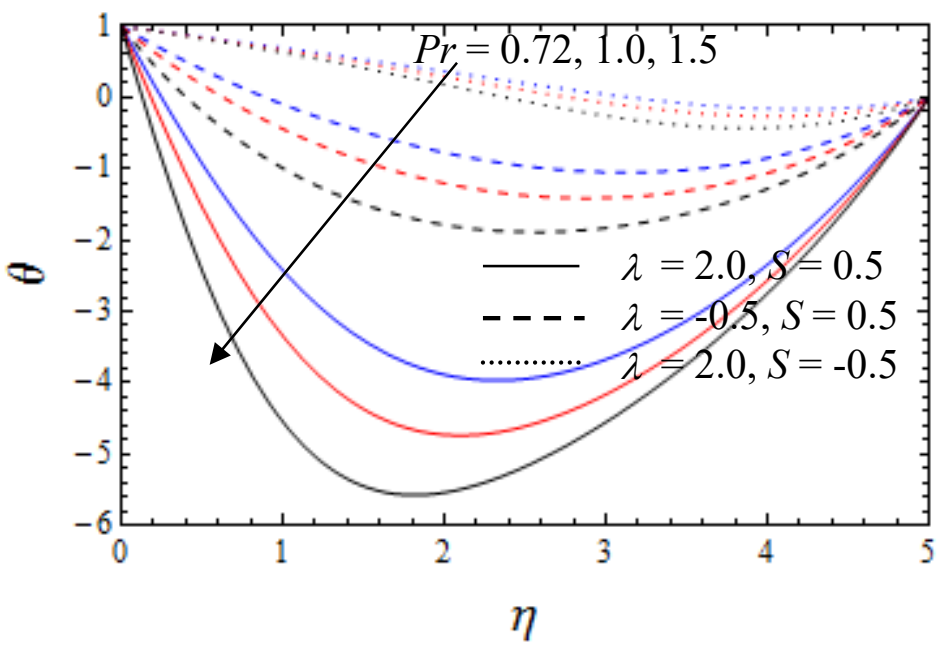

Fig. 5 Temperature profiles for $P r$

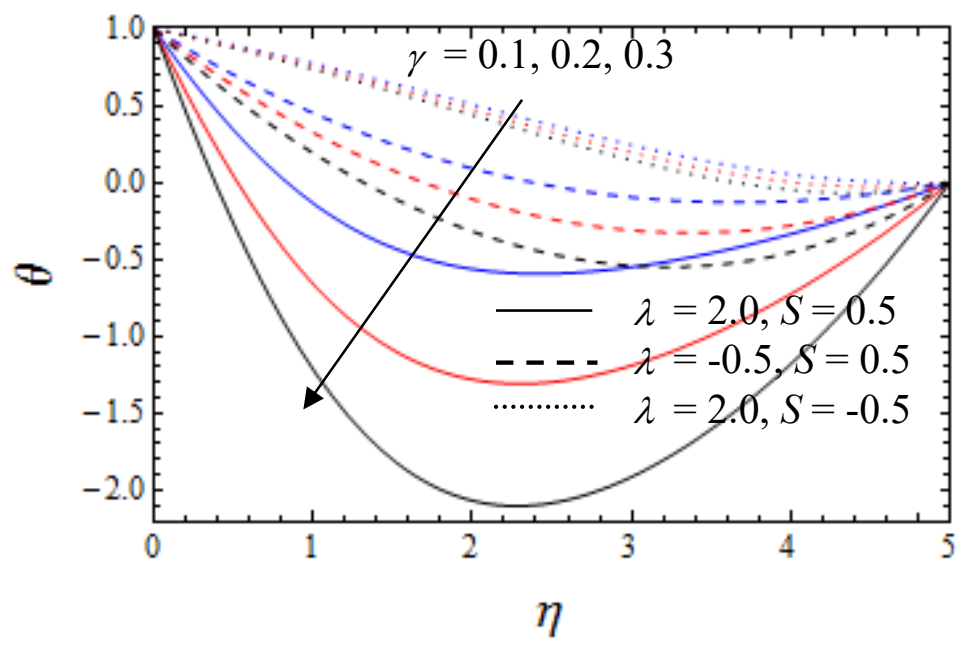

Fig. 6 Temperature profiles for $\gamma$

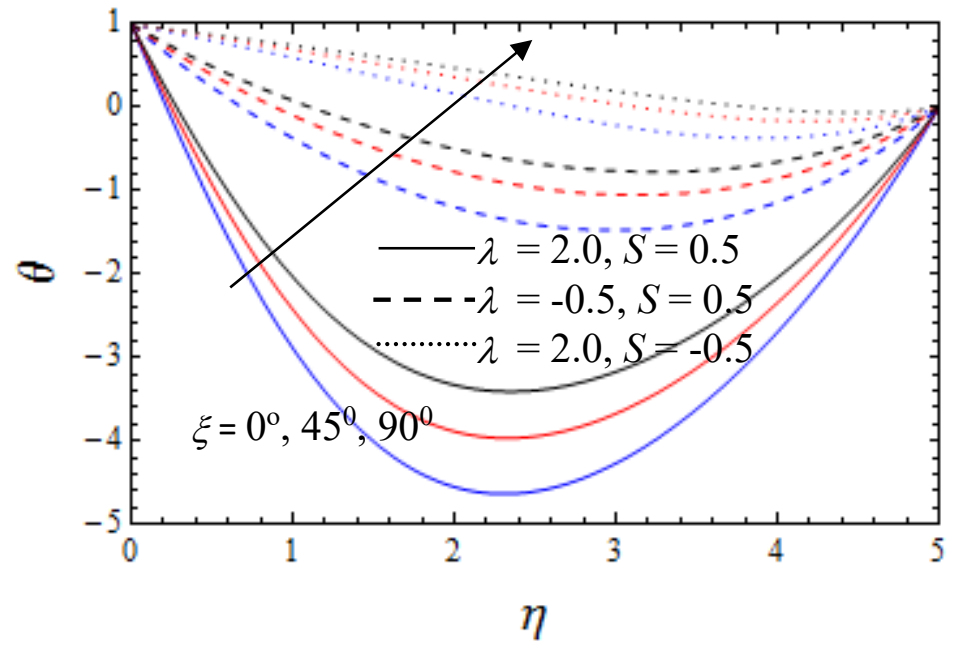

Fig. 7 Temperature profiles for $\xi$

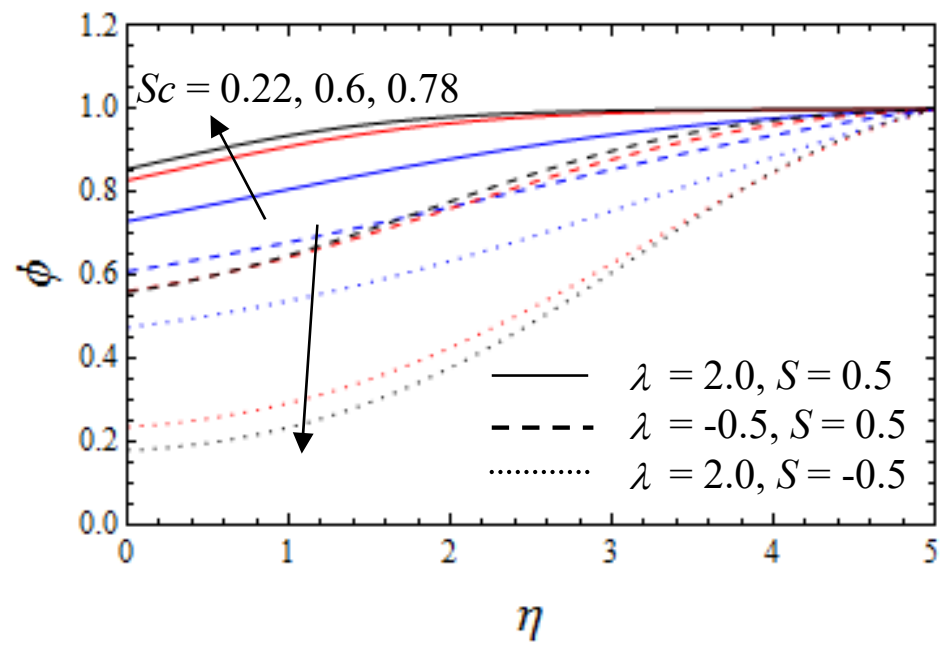

Fig. 8 Concentration profiles for $S c$

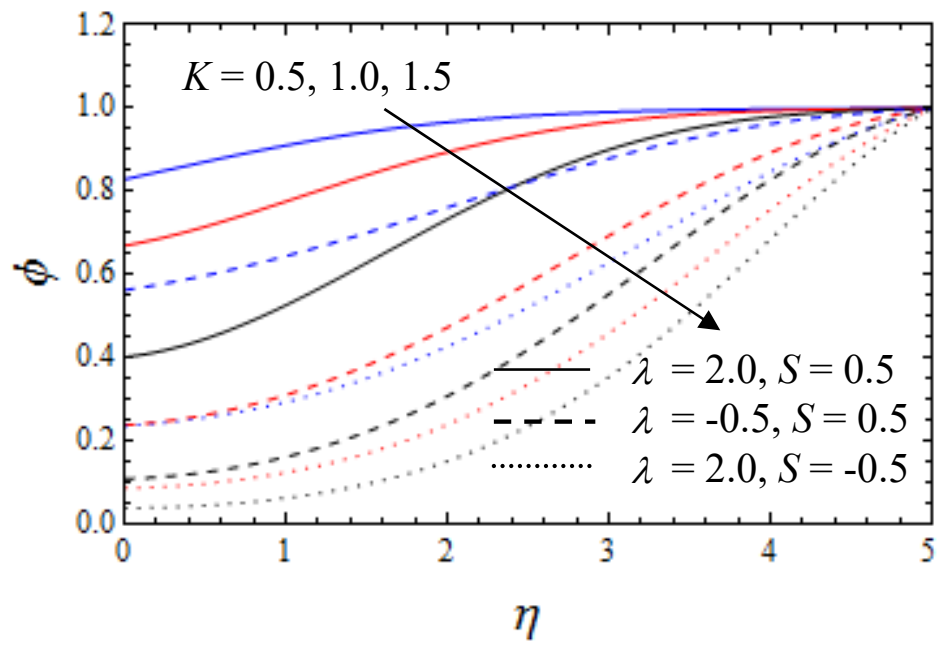

Fig. 9 Concentration profiles for $K$ 


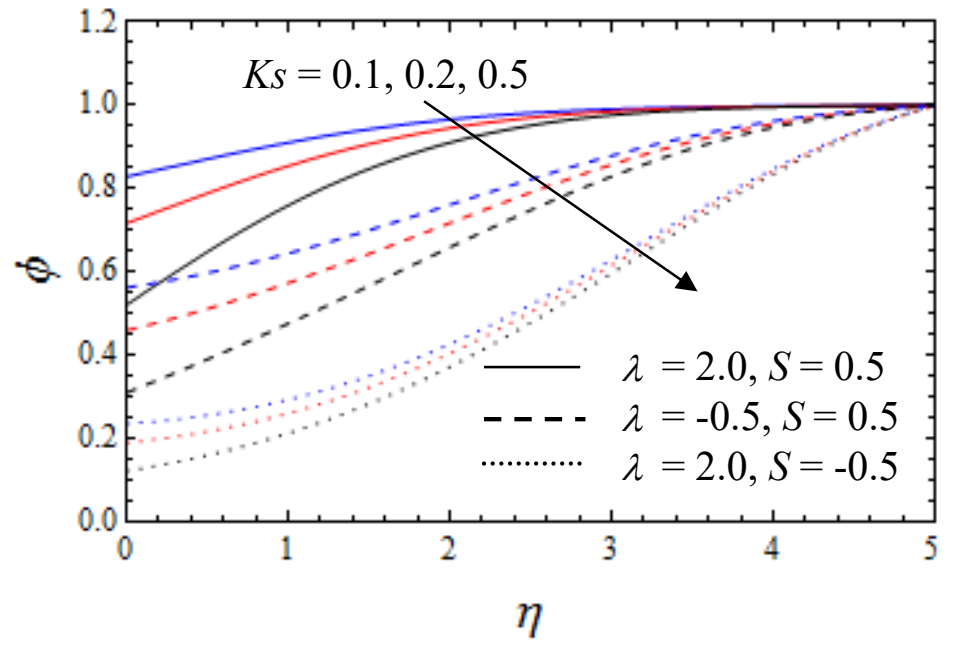

Fig. 10 Concentration profiles for $K_{s}$

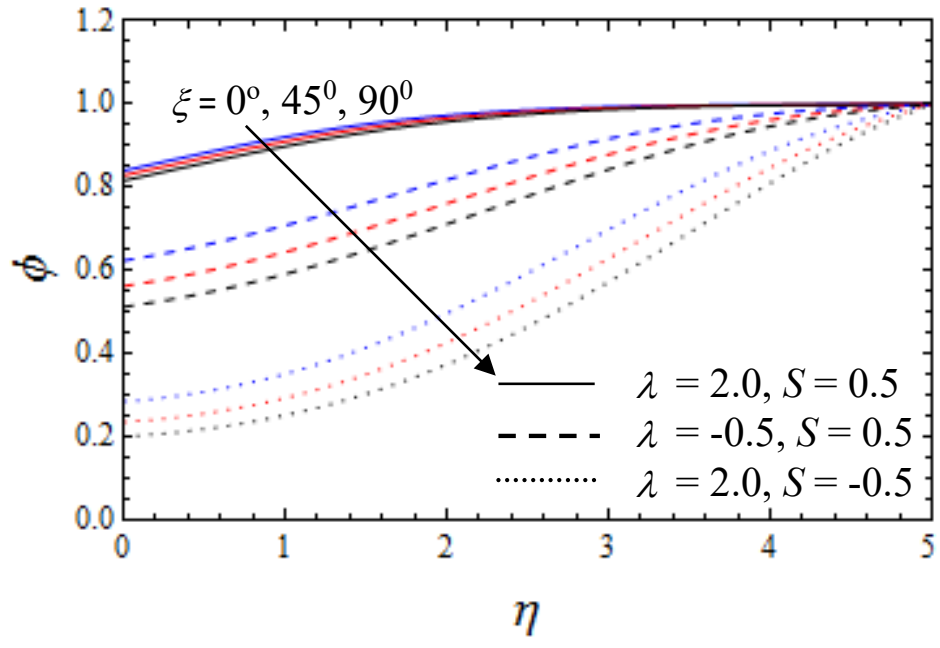

Fig. 11 Concentration profiles for $\xi$

Table 1 The values of skin friction coefficient and Nusselt number.

\begin{tabular}{|c|c|c|c|c|c|c|c|c|c|c|c|}
\hline$\beta$ & $M$ & $\xi$ & $P r$ & $\gamma$ & $\lambda$ & $S_{v}$ & $K s$ & $K$ & $S$ & $\left(1+\frac{1}{\beta}\right) f^{\prime \prime}(0)$ & $-\theta^{\prime}(0)$ \\
& & & & & & & & & & & \\
\hline 0.5 & 0.5 & $\pi / 4$ & 0.72 & 0.5 & 0.5 & 0.5 & 0.1 & 0.5 & 0.5 & -0.232394 & 2.763020 \\
\hline 1.0 & 0.5 & $\pi / 4$ & 0.72 & 0.5 & 0.5 & 0.5 & 0.1 & 0.5 & 0.5 & -0.290705 & 2.587100 \\
\hline 0.5 & 1.0 & $\pi / 4$ & 0.72 & 0.5 & 0.5 & 0.5 & 0.1 & 0.5 & 0.5 & -0.335802 & 2.420110 \\
\hline 0.5 & 0.5 & $\pi / 2$ & 0.72 & 0.5 & 0.5 & 0.5 & 0.1 & 0.5 & 0.5 & -0.335800 & 2.420110 \\
\hline 0.5 & 0.5 & $\pi / 4$ & 1.0 & 0.5 & 0.5 & 0.5 & 0.1 & 0.5 & 0.5 & -0.232394 & 3.831060 \\
\hline 0.5 & 0.5 & $\pi / 4$ & 0.72 & 1.0 & 0.5 & 0.5 & 0.1 & 0.5 & 0.5 & -0.232394 & 5.308170 \\
\hline 0.5 & 0.5 & $\pi / 4$ & 0.72 & 0.5 & 1.0 & 0.5 & 0.1 & 0.5 & 0.5 & -0.894464 & 3.521560 \\
\hline 0.5 & 0.5 & $\pi / 4$ & 0.72 & 0.5 & 0.5 & 1.0 & 0.1 & 0.5 & 0.5 & -0.164150 & 2.673750 \\
\hline 0.5 & 0.5 & $\pi / 4$ & 0.72 & 0.5 & 0.5 & 0.5 & 0.2 & 0.5 & 0.5 & -0.232394 & 2.763020 \\
\hline 0.5 & 0.5 & $\pi / 4$ & 0.72 & 0.5 & 0.5 & 0.5 & 0.1 & 1.0 & 0.5 & -0.232394 & 2.763020 \\
\hline 0.5 & 0.5 & $\pi / 4$ & 0.72 & 0.5 & 0.5 & 0.5 & 0.1 & 0.5 & 1.0 & -0.209097 & 5.433510 \\
\hline
\end{tabular}

\section{REFERENCES}

Abdul Hakeem A.K., Renuka, P., Vishnu Ganesh, N., Kalaivanan, R. and Ganga, B., 2016, "Influence of Inclined Lorentz Forces on Boundary Layer Flow of Casson Fluid over an Impermeable Stretching Sheet With Heat Transfer," Journal of Magnetism and Magnetic Materials, 401, 354-361.

https://doi.org/10.1016/i.jmmm.2015.10.026

Akbar N.S., 2015, "Influence of Magnetic Feld on Peristaltic Flow of a Casson Fuid in an Asymmetric Channel: Application in Crude Oil Refinement," J. Magn. Mater, 378(15), 463-468. https://doi.org/10.1016/j.jmmm.2014.11.045

Bala Anki Reddy P., and Bhaskar Reddy N., 2011, "Thermal Radiation Effects on Hydromagnetic Flow Due to an Exponentially Stretching Sheet," Int. J. Appl. Math. Comput, 3(4), 300-306.

https://doi.org/10.0000/ijamc.2011.3.4.125

Bala Anki Reddy P., 2016, "Magnetohydrodynamic Flow of a Casson Fluid over an Exponentially Inclined Permeable Stretching Surface with
Thermal Radiation and Chemical Reaction," Ain Shams Engineering Journal, 7, 593-602.

https://doi.org/10.1016/j.asej.2015.12.010

Bala Anki Reddy P., Suneetha S., and Bhaskar Reddy N., 2016, "Numerical Study of MHD Boundary Layer Slip Flow of a Maxwell Nanofluid over an Exponentially Stretching Surface with Convective Boundary Condition," Propulsion and Power Research, (Accepted).

Bhattacharyya K., Hayat T., and Alsaed A.,2013, "Analytic Solution for MHD Boundary Layer Flow of Casson Fluid over a Stretching/Shrinking Sheet with Wall Mass Transfer," Chinese Physics $B, 22,024702$.

https://doi.org/10.1088/1674-1056/22/2/024702

Bird, B.R., Stewart, W.E., Lightfoot, E.N, 2006, Transport Phenomena, Revised $2^{\text {nd }}$ Ed., John Wiley and Sons, New York.

Casson N., 1959, "a Flow Equation for Pigment-Oil Suspensions of The Printing Ink Type Rheology of Disperse Systems," Pergamon Press, London, UK. 
Chaudhary M.A., and Merkin J.H., 1995, “A Simple Isothermal Model For Homogeneous-Heterogeneous Reactions In Boundary-Layer Flow. I Equal Diffusivities," Fluid Dyn, 16, 311-333. https://doi.org/10.1016/0169-5983(95)00015-6

Christov C.I., 2009, "on Frame Indifferent Formulation of the MaxwellCattaneo Model of Finite- Speed Heat Conduction," Mechanics Research Communication, 36, 481-486.

https://doi.org/10.1016/j.mechrescom.2008.11.003

Crane L.J., 1970, "Flow Past a Stretching Plate," Z Angew. Math. Phys, 21, 645-647.

Cussler E.L., 1998, "Diffusion Mass Transfer in Fluid Systems," Cambridge University Press, London, UK.

Dash R.K., Mehta K.N. and Jayaraman G., 1996, "Casson Fluid Flow in a Pipe Filled with a Homogeneous Porous Medium," Int $J$ Eng Sci, 34(10), 1145-56.

https://doi.org/10.1016/0020-7225(96)00012-2

Dong Y., Cao B.Y., and Guo Z.Y., 2011, "Generalized Heat Conduction Laws Based on Thermo Mass Theory and Phonon Hydrodynamics," Journal of Applied Physics, 110, 063504-6.

https://doi.org/10.1063/1.3634113

Hassan M., Pathak M., and Khan M.K., 2013, "Natural Convection of Viscoplastic Fluids in a Square Enclosure," J. Heat Transfer, 135(12), 122501-12.

https://doi.org/10.1115/1.4024896

Hayat, T., Imtiaz, M., Alsaedi, A. and Almezal, S.,2016, "on CattaneoChristov Heat Flux in MHD Flow of Oldroyd-B Fluid with Homogeneous-Heterogeneous Reactions," J. Mag. Mag. Mat.,401,296303.

https://doi.org/10.1016/j.jmmm.2015.10.039

Hayat T., Farooq M., Alsaedi A., and Falleh Al-Solamy., 2015, "Impact of Cattaneo-Christov Heat Flux in the Flow over a Stretching Sheet with Variable Thickness," AIP Advances,5,087159-12. http://dx.doi.org/10.1063/1.4929523

Khalid A., Ilyas Khan., Arshad Khan., and Sharidan Shafie., 2015, "Unsteady MHD Free Convection flow of Casson Fluid Past over an Oscillating Vertical Plate Embedded in a Porous Medium," Engineering Science and Technology, an International Journal, 18, 309-317. https://doi.org/10.1016/j.jestch.2014.12.006

Khan, Fakhar K., and Anwar M.I., 2012, "Hydromagnetic Rotating Flows of an Oldroyd-B Fluid in a Porous Medium Special Top," Rev Porous Media, 3, 189-95.

https://doi.org/10.1615/SpecialTopicsRevPorousMedia.v3.i1.80

Khan, Farhad A., Sharidan S., and Qasim M., 2014, "Unsteady Free Convection Flow in a Walter-B- Fluid And Heat Transfer Analysis," Malay. Math Sc. Soc, 37, 437-448.

Kirubhashankar C.K., Ganesh S., and Mohamed Ismail A., 2015, "Casson Fluid Flow and Heat Transfer over an Unsteady Porous Stretching Surface," Applied Mathematical Sciences, 9(7), 345-351. http://dx.doi.org/10.12988/ams.2015.411988

Kleppe J. and Marner W.J., 1972, "Transient Free Convection in a Bingham Plastic on a Vertical Flat Plate," J. Heat Transfer, 94(4), 371376.
Krishnendu Bhattacharyya., 2013, "Boundary Layer Stagnation- Point Flow of Casson Fluid And Heat Transfer Towards a Stretching/Shrinking Sheet," Frontiers in Heat and Mass Transfer, 4, 023003-1-9.

http://dx.doi.org/10.5098/hmt.v4.2.3003

Malik M.Y., Mair Khan., Salahuddin T., and Imad Khan., 2016, "Variable Viscosity And MHD Flow in Casson Fluid with CattaneoChristov Heat Flux Model: Using Keller Box Method," Engineering Science and Technology, an International Journal, 19(4), 1985-1992. https://doi.org/10.1016/j.jestch.2016.06.008

Mariam Sheikh., and Zaheer Abbas., 2015, "HomogeneousHeterogeneous Reactions in Stagnation Point Flow of Casson Fluid Due to a Stretching/Shrinking Sheet with Uniform Suction and Slip Effects," Ain Shams Engineering Journal, 1-8.

https://doi.org/10.1016/j.asej.2015.09.010

Mukhopadhyay Swati., and Rama Subba Reddy G., 2014, "Diffusion of Chemically Reactive Species of a Casson Fluid Flow over an Exponentially Stretching Surface," Thermal Energy and Power Engineering, 3, 216-221.

Mustafa M., 2015, "Cattaneo-Christov Heat Flux Model for Rotating Flow and Heat Transfer of Upper-Convected Maxwell Fluid," AIP Advances, 5, 047109-10.

http://dx.doi.org/10.1063/1.4917306

Mustafa M., and Junaid Ahmad Khan., 2015, "Model For Flow of Casson Nanofluid Past a Non-Linearly Stretching Sheet Considering Magnetic Field Effects," AIP Advances, 5, 077148. http://dx.doi.org/10.1063/1.4927449

Mustafa M., Hayat T., Pop I., and Aziz., 2011, "Unsteady Boundary Layer Flow of a Casson Fluid Due to an Impulsively Started Moving Flat Plate," Heat Transfer Asian Research, 40(6), 563-576.

http://doi.org/10.1002/htj.20358

Nadeem S., Ul Haq R., and Lee C., 2012, "MHD Flow of a Casson Fluid over an Exponentially Shrinking Sheet," Sci. Iran 19(6), 1550-3. https://doi.org/10.1016/j.scient.2012.10.021

Olajuwon B., 2009, "Flow And Natural Convection Heat Transfer in a Power Law Fluid Past a Vertical Plate with Heat Generation," Int. J. Nonlinear Sci, 7(1), 50-56.

Pramanik S., 2015, "Casson Fluid Flow And Heat Transfer Past an Exponentially Porous Stretching Surface in Presence of Thermal Radiation," Ain Shams Engineering Journal, .5(1), 205- 212.

https://doi.org/10.1016/j.asej.2013.05.003

Rabia Malik., Masood Khan., and Muhammad Mushtaq., 2016, "Cattaneo-Christov Heat Flux Model For Sisko Fluid Flow Past a Permeable Non-Linearly Stretching Cylinder," Journal of Molecular Liquids, 222, 430-434.

https://doi.org/10.1016/j.molliq.2016.07.040

Sachin Shaw., Peri K Kameswaran., and Precious Sibanda., 2013, "Homogeneous-Heterogeneous Reactions in Micropolar Fluid Flow From a Permeable Stretching or Shrinking Sheet in a Porous Medium," Boundary Value Problems, 77.

http://doi.org/10.1186/1687-2770-2013-77

Srinivas S., Bala Anki Reddy P., and Prasad BSRV., 2014, "Effects of Chemical Reaction And Thermal Radiation on MHD Flow over an Inclined Permeable Stretching Surface with Non-Uniform Heat 
Source/Sink: an Application to the Dynamics of Blood Flow," Journal of Mechanics in Medicine and Biology, 14(5), 1450067.

https://doi.org/10.1142/S0219519414500675

Sumalatha C., and Bandari S., 2015, "Effects of Radiations and Heat Source/Sink on a Casson Fluid Flow over Nonlinear Stretching Sheet," World Journal of Mechanics, 5, 257-265.

https://doi.org/10.4236/wjm.2015.5120244

Swati Mukhopadhyay., 2013, "Casson Fluid Flow And Heat Transfer over a Nonlinearly Stretching Surface," Chin. Phys. B, 22(7), 0747015.https://doi.org/10.1088/1674-1056/22/7/074701

Swati Mukhopadhyay., Prativa Ranjan De., Krishnendu Bhattacharyya., and Layek G.C., 2013, "Casson Fluid Flow over Unsteady Stretching Surface," Ain Shams Engineering Journal, 4, 933-938.

https://doi.org/10.1016/j.asej.2013.04.004

Tasawar Hayat., Sumaira Qayyum., Maria Imtiaz., and Ahmed Alsaedi., 2016, "Three-Dimensional Rotating Flow of Jeffrey Fluid For Cattaneo-Christov Heat Flux Model," AIP Advances, 6, 025012. http://dx.doi.org/10.1063/1.4942091
Tasawar Hayat, Mehwish Javed, MariaImtiaz, and AhmedAlsaedi.,2018, " Effect of Cattaneo-Christov Heat Flux on Jeffrey Fluid Flow with Variable Thermal Conductivity," Results in Physics 8,341-351.

https://doi.org/10.1016/j.rinp.2017.12.007

Zakaria M.N., Hussanan A., Khan I., and Shafie S., 2013, "The Effects of Radiation on Free Convection Flow with Ramped Wall Temperature in Brinkman Type Fluid," J. Teknol, 62(3), 33-39.

http://dx.doi.org/10.11113/jt.v62.1886

Muhammad Ijaz Khan, Muhammad Waqas, Tasawar Hayat, Muhammad Imran Khan and Ahmed Alsaedi, 2017, "Chemically Reactive Flow of Upper-Convected Maxwell Fluid with CattaneoChristov Heat Flux Model," J Braz. Soc. Mech. Sci.Eng. 39, 24714578.https://doi.org/10.1007/s40430-017-0915-5

Imad Khan, Malik M.Y., Arif Hussain., and Salahuddin T., 2017, "Effect of Homogenous-Heterogeneous Reactions on MHD Prandtl Fluid Flow over a Stretching Sheet," Results in Physics, 7, 4226-4231. https://doi.org/10.1016/j.rinp.2017.10.052. 